\title{
Register autorum ad Vol. 16
}

(B) = Buchbeprechung - Book Review - Livre nouveau. $(\mathrm{R})=$ Referat - Review - Revue

Register autorv

(B) = Buchbeprechung - Book Review - Livre

Aleksandrowicz, J., 42

Alvarez, A. M. Romero, v. Romero

Asboe-Hansen, G., and Kaalund-Jørgen-sen, O., 273

Begemann, H., und Harwerth, H.-G., 280 ff. (R), 347 ff. (R)

Bergna, L.J., and Pavlovsky, A., 247

Beyreder, J., and Rieder, H., 299

Bianchini, E., v. Fieschi, A.

Blaser, E., Cretin, P., und Stampfli, K., 407

Böttiger, L. E., and Hellstròm, O., 329

Braunsteiner, H., Fellinger, K., und Pakesch, F., 322

Brücher, PL, und Nathan, S., 30

Brücher, H., v. Warnakoff, H.

Cambiaggi, G., v. Fieschi, A.

Cheli, R., and Dianzani, M. U., 37

Cretin, P., v. Blaser, E.

De Nicola, v. Nicola

De Vries, v. Vries

Dianzani, M. U., v. Cheli, R.

Fellinger, K., v. Braunsteiner, H.

Fieschi, A., Bianchini, E., Cambiaggi, G., Sacchetti, C, and Salvidio, E., 126

Frick, P. G., 11

Gassmann, W., und Schneeweiss, J., 396

Gelin, G., 297 (B)

Greig, H. B. W., 171

Gross, R., Heuer, J., und Solth, K., 147

Guidi, G., v. Scardigli, G.

Haanen, C. A. M., 363

Harwerth, H.-G., v. Begemann, H.

Haschen, R.J., 235

Heckner, F., 1

Hellstròm, O., v. Böttiger, L. E.

Heuer, J., v. Gross, R.

Heuser, G., v. Selye, H.

Jürgens, J., 181

Kaalund-Jørgensen, O., v.Asboe-Hansen,G. 
Kaulla, K. N. v., 315

Köster, Erika, v. Lennert, K.

Roller, F., 46 ff. (R)

Koshiishi, Y., v. Otsuka, S.

Krejnis, E., v. Shafrir, E.

Kubota, Chrysanthia, Schwartz, St. O., and Putnam, F. W., 105

Künzer, W., Schütz, A., und Schütz, E., 137

Kumai, K., v. Otsuka, S.

Lennert, K., Köster, Erika, und Martin, H., 255

Malý, V., v. Polák, H.

Margulies, M., v. Romero Alvarez, A. M.

Martin, H., v. Lennert, K.

Meurman, L., and Odeblad, E., 400

Meyer, G., v. Romero Alvarez, A. M.

Moeschlin, S., 170 (B)

Müller, W., v. Weinreich, J.

Naruto, K., v. Otsuka, S.

Nathan, S., v. Brücher, H.

Nicola, P. de, 298 (B)

Odeblad, E., v. Meurman, L.

Oechslin, R.J., 214

Otsuka, S., Koshiishi, Y., Naruto, K., and Kumai, K., 199

Pakesch, F., v. Braunsteiner, H.

Pavlovsky, A., v. Bergna, L. J.

Polák, H., and Poláková, Kvëta, (and Malý, V.), 385

Poláková, Kvëta, v. Polák, H.

Putnam, F. W., v. Kubota, Chrysanthia

Ramot, Bracha, and Singer, K., 158

Rieder, H., v. Beyreder, J.

Rohr, K., 297 (B)

Romero Alvarez, A. M., Meyer, G., Margulies, M., y Vilaseca, G., 170 (B)

Rosenow, G., 170 (B); 298 (B)

Sacchetti, C, v. Fieschi, A.

Salvidio, E., v. Fieschi, A

Scardigli, G., and Guidi, G., 338

Schneeweiss, J., v. Gassmann, W.

Schütz, A., v. Künzer, W.

Schütz, E., v. Künzer, W.

Schwartz, St. O., v. Kubota, Chrysanthia

Selye, H., and Heuser, G. (Editores), 298 (B)

Shafrir, E., Vries, A. de, and Krejnis, E., 204

Singer, K., v. Ramot, Bracha

Snapper, I., 298 (B)

Solth, K., v. Gross, R.

Stampfli, K., v. Blaser, E.

Sturgis, C. C, 170 (B)

Tocantins, L. M. (Editor), 298 (B)

Videbaek, A., 411 ff. (R)

Vilaseca, G., v. Romero Alvarez, A. M.

Vries, A. de, v. Shafrir, E.

Warnakoff, H., und Brücher, H., 116

Weinreich, J., und Müller, W., 376 